\title{
GROUPS WITH A SUBLINEAR ISOPERIMETRIC INEQUALITY
}

\author{
Michael Batty
}

Abstract We give a proof that if a finitely presented group $G$ admits a presentation with a sublinear isoperimetric inequality, then $G$ is either free or finite.

\section{Introduction}

Let $G=\langle S \mid R\rangle$ be a finitely generated group. Then a word in $F(S)$, the free group on $S$, is equal to the identity in $G$ if and only if there exist words $u_{i}$ in $S$ for $1 \leq i \leq n$ such that

$$
w=\prod_{i=1}^{n} u_{i} r_{i} u_{i}^{-1} \text { as reduced words }
$$

where for all $i$ with $1 \leq i \leq n$ either $r_{i} \in R$ or $r_{i}^{-1} \in R$.

Definition 1.1 With $G$ as above, let $w$ be a word in $S$ which is equal to the identity in $G$. Then the area of $w, A(w)$ is defined to be

$$
\min \left\{n \in \mathbb{N} \mid \exists \text { an equality } w=\prod_{i=1}^{n} u_{i} r_{i} u_{i}^{-1} \text { in } F(S)\right\} .
$$

We do not work with this definition of area but rather with a more geometric formulation.

Definition 1.2 A map is a finite, planar, oriented, connected and simply connected combinatorial 2-complex.

Let $M$ be a map with edge set $E(M)$. If $e=\left(v_{1}, v_{2}\right) \in E(M)$ then we write $\tilde{e}$ for the edge $\left(v_{2}, v_{1}\right)$. 
Definition 1.3 A paired alphabet is a finite set $S$ together with an involution $f: S \rightarrow S$. We usually write $f(s)=s^{-1}$.

For example, an inverse closed set of generators of a group is a paired alphabet, where the involution is the group inverse.

Definition A diagram over a paired alphabet $S$ is a triple $(M, S, l)$ where $M$ is a map, $S$ is a paired alphabet and $l: E(M) \rightarrow$ $S$ satisfies $l(\tilde{e})=(l(e))^{-1}$ for all $e \in E(M)$. If $e \in E(M)$ then $l(e)$ is called the label of $e$.

When we refer to a path in a graph $X$ we mean a finite sequence of adjacent edges. If the sequence of terminal vertices of edges consists of distinct vertices then we call the path simple. A loop is a path such that the terminal vertex of the final edge equals the initial vertex of the first. Thus a simple loop is a loop which is simple as a path. If $X$ is the 1-skeleton of a diagram $(M, S, l)$ and $p=e_{1}, \ldots, e_{n}$ is a path in $X$ we define its label $l(e)$ to be the word $l\left(e_{1}\right) \cdots l\left(e_{n}\right)$ in $S$. If $f$ is a face of $M$ we denote its boundary loop by $\partial f$ and write $l(f)$ for $l(\partial f)$.

Definition 1.5 Let $G=\langle S, R\rangle$ be a finitely presented group where $S$ is an inverse closed generating set for $G$. A van Kampen diagram over $G$ is a diagram $M$ over $S$ such that for all faces $f$ of $M, l(f)=r^{ \pm 1}$ for some relator $r \in R$. The area of such a diagram is the number of its faces.

The hypotheses on a map $M$ ensure that its boundary $\partial M$ is a loop. We write $l(M)$ for $l(\partial M)$. If $G=\langle S \mid R\rangle$ is a group presentation and $w$ is a word in $S$ then we write $\bar{w}$ for the element of $G$ represented by $w$.

Lemma 1.6 (van Kampen) Let $G=\langle S \mid R\rangle$ be a finitely presented group and let $w$ be a word in $S$. Then $\bar{w}=1_{G}$ if and only if there exists a Van Kampen diagram $M$ over $G$ with $l(M)=w$. Moreover $A(w)$ is equal to the least area of a van Kampen diagram for $w$.

Proof: See [7].

Definition 1.7 Let $G=\langle S \mid R\rangle$ be a finitely presented group. Then the Dehn function $D$ of $G$ with respect to $S$ and $R$ is the 
function $D: \mathbb{N} \rightarrow \mathbb{N}$ given by

$$
D(n)=\max \left\{A(w) \mid \bar{w}=1_{G} \text { and } l(w) \leq n\right\} .
$$

We say that $G=\langle S \mid R\rangle$ satisfies a linear isoperimetric inequality if its Dehn function is $O(n)$, i.e. there exists $C \geq 0$ such that for all $n \in \mathbb{N}, D(n) \leq C n$. If

$$
\lim _{n \rightarrow \infty}\left(\frac{D(n)}{n}\right)=0
$$

then we say that $G$ satisfies a sublinear isoperimetric inequality and if

$$
\lim _{n \rightarrow \infty}\left(\frac{D(n)}{n^{2}}\right)=0
$$

then we say that $G$ satisfies a subquadratic isoperimetric inequality. If $G$ is a finitely presented group then it is well known that the following are equivalent.

1. $G$ is hyperbolic in the sense of Gromov [3].

2. $G$ satisfies a linear isoperimetric inequality.

3. $G$ satisfies a subquadratic isoperimetric inequality.

The proof that the first statement is equivalent to the second can be found in [6]. The second clearly implies the third. The fact that a subquadratic isoperimetric inequality implies a linear one is originally due to Gromov [3] and can be found in [2], [4] and [5]. In particular we see that the satisfaction of a sublinear isoperimetric inequality is invariant under quasi-isometry.

\section{Quasi-Trees}

A graph $X$ is said to be of bounded valency if there exists an integer $N$ such that the valency of every vertex of $X$ is at most $N$.

Definition 2.1 Let $Q$ be a connected graph of bounded valency. We call $Q$ a $K$-quasi-tree if every simple loop in $Q$ has length at most $K$. If there exists a non-negative integer $K$ for which $Q$ is a $K$-quasi-tree then we call $Q$ a quasi-tree. 
Theorem 2.2 A finitely generated group $G$ acts freely on a quasitree if and only if $G$ is isomorphic to a free product of free groups and finite groups.

A proof is given in [1].

\section{The Main Result}

Let $G$ be a group with a finite generating set $S$. We write $\Gamma_{S}(G)$ for the Cayley graph of $G$ with respect to $S$. Suppose that we have a sublinear isoperimetric inequality amongst the simple loops in $\Gamma_{S}(G)$ (i.e. in formation of the Dehn function we only consider simple loops). In this situation we say that $G$ satisfies a sublinear simple isoperimetric inequality.

Proposition 3.1 If a finitely presented group $G$ satisfies a sublinear simple isoperimetric inequality, then there is a bound on the length of simple loops in its Cayley graph.

Proof: Suppose that in the Cayley graph $\Gamma_{S}(G)$ of $G$ with respect to some finite presentation $\langle S \mid R\rangle$ there is satisfied a sublinear simple isoperimetric inequality. Let $K$ be the maximum length of the relators. Assume that $R$ is not empty (if it is then the theorem follows easily), so that $K \geq 1$. If a simple loop $\Lambda$ in $\Gamma_{S}(G)$ has length $l$ then the number of relators we require to fill $\Lambda$ is at least the next integer after $\frac{l}{K}$. So unless there is a bound on the length of simple loops in $\Gamma_{S}(G)$, the best bound below for the isoperimetric inequality of $G$ is at least a linear function.

Corollary 3.2 A finitely presented group $G$ admits a sublinear simple isoperimetric inequality for some finite presentation if and only if $G$ is quasi-free.

Proof: If $G$ is a quasi-free group, then with respect to the standard generating set there is a bound on the length of simple loops in its Cayley graph. So $G$ clearly satisfies a sublinear simple isoperimetric inequality.

Conversely, by Proposition 3.1, the sublinear simple isoperimetric inequality gives us a bound on the length of simple loops in $\Gamma_{S}(G)$. Hence $\Gamma_{S}(G)$ is a quasi-tree upon which $G$ acts freely. Now the result follows by Theorem 2.2. 
Note that the property of whether or not a group admits a sublinear isoperimetric inequality is not invariant under quasi-isometry. For example, $\mathbb{Z}$ trivially satisfies a sublinear isoperimetric inequality with respect to the standard generating set. However, with the standard generating set the group $\mathbb{Z} \oplus \mathbb{Z}_{3}$, which is quasi-isometric to $\mathbb{Z}$, does not.

In what follows we use the notation $i(p)$ and $t(p)$ to denote the initial and terminal vertices of a path $p$ in a graph $X$. We also give each loop $L$ in $X$ a preferred orientation and if $v_{1}$ and $v_{2}$ are vertices of $L$ write $L\left(v_{1}, v_{2}\right)$ for the path obtained when travelling around $L$ from $v_{1}$ to $v_{2}$ in a positive direction.
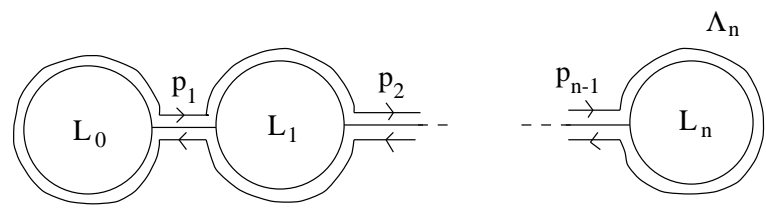

A loop with linear area.

Theorem 3.3. If a group $G$ has a finite presentation $\langle S \mid R\rangle$ with respect to which $G$ satisfies a sublinear isoperimetric inequality then $G$ is either free or finite.

Proof: In particular, $G$ satisfies a sublinear simple isoperimetric inequality. Hence by Corollary $3.2, \Gamma_{S}(G)$ is a quasi-tree and $G$ is quasi-free. Now suppose that there is a nontrivial finite group $H$ which is a free factor of $G$. Let $H_{0}$ be the subgraph of $\Gamma_{S}(G)$ induced by the vertex set of $H$. Either $G$ is finite or $\Gamma_{S}(G)$ contains infinitely many copies of $H_{0}$. Let $M$ be the maximum length of a relator in $R$. We may choose a loop $L$ of strictly positive area in $H$ and copies $H_{0}=H, H_{1}, \ldots, H_{n}, \ldots$ of $H$ containing copies $L_{0}=L, L_{1}, \ldots, L_{n}, \ldots$ of $L$ in such a way that there are paths $p_{1}, \ldots, p_{n}, \ldots$, all of the same length, where for each $j, p_{j}$ goes from $L_{j-1}$ to $L_{j}$ as in figure 1 . Furthermore, we may choose these 
such that $d\left(i\left(p_{j}\right), t\left(p_{j}\right)\right)>M$ for all $j$. Let $\Lambda_{n}$ be the loop

$$
\begin{array}{r}
L_{0}\left(1, i\left(p_{1}\right)\right) * p_{1} * L_{1}\left(t\left(p_{1}\right), i\left(p_{2}\right)\right) * p_{2} * \cdots \\
* p_{n-1} * L_{n} * p_{n-1}^{-1} * \cdots * p_{2}^{-1} * L_{1}\left(i\left(p_{2}\right), t\left(p_{1}\right)\right) * p_{1}^{-1} * L_{0}(i(p), 1) .
\end{array}
$$

Let $N=A(L)$. Then since $i\left(p_{j}\right)$ and $t\left(p_{j}\right)$ are cut points of $\Gamma_{S}(G)$ and the endpoints of the paths $p_{j}$ are sufficiently far apart, $A\left(\Lambda_{n}\right)=N n$. Thus $G$ satisfies an isoperimetric inequality which is at least linear, a contradiction. Hence $G$ is free.

\section{Acknowledgements}

I would like to thank Martin Bridson, David Epstein and Derek Holt for useful conversations leading to this paper.

\section{References}

[1] M. Batty, A splitting theorem for groups acting on quasi-trees, in preparation.

[2] B. H. Bowditch, A short proof that a subquadratic isoperimetric inequality implies a linear one, Michigan Math J. 42 (1995) 103-107.

[3] M. Gromov, Hyperbolic groups, in Essays in Group Theory, ed. by S. Gersten, 75-263. MSRI Publications No. 8, Springer-Verlag, 1988.

[4] A. Ol'Shanskii, Hyperbolicity of groups with a subquadratic isoperimetric inequality, Int. J. Alg. and Comp. 1 (1991), 281-289.

[5] P. Papasoglu, On the sub-quadratic isoperimetric inequality, in Proceedings of the Ohio-State Program in Geometric Group Theory, ed. by R. Charney and M. Davis.

[6] H. Short, Notes on word hyperbolic groups in Group Theory from the Geometrical Viewpoint, ed. by E. Ghys, A. Haefliger and A. Verjovsky, 3-63. World Scientific: Singapore, 1991.

[7] E. R. van Kampen, On some lemmas in the theory of groups, Amer. J. Math. 55 (1933), 268-273.

Michael Batty

Department of Mathematics National University of Ireland Galway

email: michael.batty@ucg.ie 